\title{
The Spectrum of the Kinematic Dynamo Operator for an Ideally Conducting Fluid
}

\author{
C. Chicone*, Y. Latushkin ${ }^{\dagger}$, and S. Montgomery-Smith \\ Department of Mathematics, University of Missouri \\ Columbia, MO 65211, USA
}

June 16, 2001

\begin{abstract}
The spectrum of the kinematic dynamo operator for an ideally conducting fluid and the spectrum of the corresponding group acting in the space of continuous divergence free vector fields on a compact Riemannian manifold are described. We prove that the spectrum of the kinematic dynamo operator is exactly one vertical strip whose boundaries can be determined in terms of the Lyapunov-Oseledets exponents with respect to all ergodic measures for the Eulerian flow. Also, we prove that the spectrum of the corresponding group is obtained from the spectrum of its generator by exponentiation. In particular, the growth bound for the group coincides with the spectral bound for the generator.
\end{abstract}

*carmen@chicone.cs.missouri.edu, supported by the NSF grant DMS-9303767;

†mathyl@mizzou1.missouri.edu, supported by the NSF grant DMS-9400518 and by the Summer Research Fellowship of the University of Missouri;

${ }^{\ddagger}$ stephen@mont.cs.missouri.edu, supported by the NSF grant DMS-9201357; Key words and phrases. Hyperbolic dynamical systems, Mather spectrum, magnetohydrodynamics, ideally conducting fluid, spectral mapping theorems, weighted composition operators. Mathematics Subject Classification. 76W05, 58F99, 58G25. 


\section{Introduction}

In this paper we give a description of the spectrum of the kinematic dynamo operator and of the corresponding group it generates for an ideally conducting fluid in the space of continuous divergence free vector fields.

Consider a steady incompressible conducting fluid with Eulerian velocity $v=v(x)$ for $x \in \mathbb{R}^{3}$ and let $\phi^{t}$ denote the corresponding flow. The kinematic dynamo equations for the induction of a magnetic field $\mathbf{H}$ by the flow has the following form:

$$
\dot{\mathbf{H}}=\nabla \times(v \times \mathbf{H})+\varepsilon \Delta \mathbf{H}, \quad \operatorname{div} \mathbf{H}=0,
$$

where $\varepsilon=\mathcal{R} e_{m}{ }^{-1}$, and $\mathcal{R} e_{m}$ is the magnetic Reynolds number (see, e.g., [15, Ch. 6]). The spectral properties of the kinematic dynamo operator $L_{\varepsilon}$, defined by (1.1), have been a subject of intensive study, in particular, in connection with the famous dynamo problem (see $[1,2,3,6,16,23]$ and references therein).

For the ideally conducting fluid, $\varepsilon=0$, these equations become:

$$
\dot{\mathbf{H}}=-(v, \nabla) \mathbf{H}+(\mathbf{H}, \nabla) v, \quad \mathbf{H}(x, 0)=\mathbf{H}_{0}(x), \quad \operatorname{div} \mathbf{H}=0 .
$$

The last equation has [15] so-called Alfven solutions

$$
\mathbf{H}(x, t)=D \phi^{t}\left(\phi^{-t} x\right) \mathbf{H}\left(\phi^{-t} x, 0\right),
$$

given by the group $\left\{e^{t L}\right\}_{t \in \mathbb{R}}$ with the generator $L=L_{0}$ that acts by the rule

$$
L: u \mapsto(u, \nabla) v-(v, \nabla) u .
$$

In the present paper the kinematic dynamo operator $L$ is considered in the following well known context. Let $v$ denote a continuous divergencefree vector field on a compact Riemannian manifold $X$ without boundary, let $\phi^{t}$ denote the flow generated by $v$ and let $D \phi^{t}(x)$ denote its differential. Consider the group $\left\{e^{t L}\right\}_{t \in \mathbb{R}}$ of push-forward operators generated by the Lie derivative $L$ in the direction $v$. This group acts on continuous sections of the tangent bundle $\mathcal{T} X$, by the rule

$$
\left(e^{t L} u\right)(x)=D \phi^{t}\left(\phi^{-t} x\right) u\left(\phi^{-t} x\right), \quad x \in X, \quad t \in \mathbb{R} .
$$


We will consider the group $\left\{e^{t L}\right\}_{t \in \mathbb{R}}$ in the space $C_{N D}(X, \mathcal{T} X)$ of the continuous vector fields with zero divergence.

Operators of the form (1.4) belong to the class of weighted composition operators. This class has been widely investigated in connection with hyperbolic dynamical systems since the celebrated paper by J. Mather [13], see also $[7,20]$, the recent papers $[4,9,10,22]$ and the detailed bibliography in [12]. The spectral properties of these operators in spaces of continuous or $p$-summable vector fields are by now well understood. However, the investigation of their spectral properties in the space of divergence-free vector fields was initiated recently by $\mathrm{R}$. de la Llave. His important work [21] inspired the present paper.

In Section 2 we prove the spectral mapping theorem in $C_{N D}(X, \mathcal{T} X)$ for the group $\left\{e^{t L}\right\}_{t \in \mathbb{R}}$, assuming that the aperiodic trajectories of $\phi^{t}$ are dense in $X$ and $\operatorname{dim} X \geq 3$. Theorems of this type for continuous and for $L_{p}$-section spaces over finite dimensional manifolds were proved in $[5,8]$ while similar results for the infinite dimensional setting were obtained in $[9,10,12]$.

The spectral mapping theorem states that the spectrum $\sigma\left(e^{t L}\right)$ of $e^{t L}$ can be obtained from the spectrum of $L$ by exponentiation. It shows, in particular, that in the space of divergence-free vector fields the spectral bound of the generator $L$ coincides with the growth bound of the group, see also Remarks 2.9-2.11 below.

Our proof of the spectral mapping theorem in Section 2 exploits the fact (cf. $[13,21]$ ) that approximative eigenfunctions of the operator (1.4) can be "localized" along trajectories of the flow. We also show that the spectrum of $L$ is invariant under vertical translations of the complex plane. The same idea can be used to analyze semigroups of weighted composition operators with general cocycles on Banach spaces, see [11].

In Section 3, we show that, for $\operatorname{dim} X \geq 2$, the spectrum $\sigma\left(e^{t L}\right), t \neq 0$ in $C_{N D}(X, \mathcal{T} X)$ is exactly one annulus centered at the origin of the complex plane. Our result generalizes a theorem in [21] where this fact was proved under the restriction that the flow is Anosov with one-dimensional spectral foliations. The possibility of relaxing the hypotheses of the theorem is discussed as an open problem in [21]. The relatively simple proofs of these facts in Section 3 can be read independently of the more difficult proofs in Section 2.

By Mather's theory (see, e.g., $[12,13,20])$, the spectrum $\sigma\left(e^{t L}\right)$ on the space $C(X, \mathcal{T} X)$ is generally the union of several disjoint annuli centered 
at the origin. Passing to the space $C_{N D}(X, \mathcal{T} X)$ dramatically changes the spectrum: the gaps, if any, between these annuli are filled. Also, using the Spectral Mapping Theorem, the spectrum of $L$ on $C_{N D}(X, \mathcal{T} X)$ is exactly one vertical strip. Since $L v=0$, this strip always contains $i \mathbb{R}$, and (1.2) does not have an exponential dichotomy. Moreover, the equation (1.2) in the space of divergence free vector fields (unlike the situation with Anosov flows, cf. [20]), does not possess a nontrivial uniform exponential dichotomy even after "moding out" the direction of the flow, see Remarks 3.7-3.8 below.

Using the results in [12], we give a description of the spectrum of $L$ in terms of the Lyapunov-Oseledets exponents over all ergodic measures on $X$, that is, we will determine the boundaries of the spectrum of $L$ via the Lyapunov exponents. This description is related to a theorem by M. Vishik [23] that states the "fast" dynamo action is impossible whenever all Lyapunov numbers are zero.

Finally, we remark that we actually prove more general results than those just mentioned. In particular, we will only assume the operator $L$ generates a $C_{0}$-group of weighted composition operators that preserves the set of $d i$ vergence free vector fields and has the form

$$
\left(T^{t} u\right)(x)=\Phi\left(\phi^{-t} x, t\right) u\left(\phi^{-t} x\right), \quad x \in X, \quad t \in \mathbb{R},
$$

where $\Phi(x, t)$ is a continuous cocycle over $\phi^{t}$, that is for $x \in X$ and $t, \tau \in \mathbb{R}$ one has $\Phi(x, t+\tau)=\Phi\left(\phi^{t} x, \tau\right) \Phi(x, t)$ and $\Phi(x, 0)=I$. Throughout the paper we use $M$ to denote the generator of the group $\left\{T^{t}\right\}, T^{t}=e^{t M}$. In particular, if $\Phi(x, t)=D \phi^{t}(x)$, then (1.5) is the push-forward operator (1.4) and $M=L$ is the Lie derivative.

We also note that our technique can be applied to obtain similar results for the space $L_{2}$.

\section{The Spectral Mapping Theorem}

In this section we will prove the Spectral Mapping Theorem. Throughout the section we suppose a smooth vector field $v$ to be given on a compact Riemannian manifold $X$ without boundary, that $v$ is divergence free with respect to the Riemannian volume and that the flow $\phi^{t}$ of $v$ satisfies the following standing hypotheses: the aperiodic trajectories of $\phi^{t}$ are dense in $X$ and $n=\operatorname{dim} X \geq 3$. Let $M$ be the generator of the group $\left\{T^{t}\right\}_{t \in \mathbb{R}}$ of weighted 
composition operators, as in (1.5), for some continuous cocycle $\Phi(x, t)$ over $\phi^{t}$. We will assume $T^{t}$ is bounded on the space $C_{N D}(X, \mathcal{T} X)$ of divergence free vector fields.

There are at least two choices for the space of continuous divergence free vector fields depending on whether the divergence is understood in the classical sense or in the sense of distributions. These spaces are defined, respectively, as follows:

$$
\begin{aligned}
C_{N D}^{0}(X, \mathcal{T} X) & =\text { closure }\left\{f \in C^{\infty}(X, \mathcal{T} X): \operatorname{div} f=0\right\} \\
C_{N D}^{1}(X, \mathcal{T} X)= & \{f \in C(X, \mathcal{T} X): \\
& \left.\int_{X}<f, \operatorname{grad} g>d \mu=0 \quad \forall g \in C^{\infty}(X, \mathbb{R})\right\} .
\end{aligned}
$$

The closure in (2.6) is taken with respect to the sup-norm while the scalar product $\langle\cdot, \cdot\rangle$ and grad in (2.7) are taken with respect to a Riemannian metric and volume on $X$. We note that the space $C_{N D}^{1}(X, \mathcal{T} X)$ is a closed subspace of $C_{N D}^{0}(X, \mathcal{T} X)$.

For a linear operator $A$ in a Banach space $E$, we will sometimes use $\sigma(A ; E)$ to denote the spectrum of $A$ on $E$ and $\sigma_{\mathrm{ap}}(A ; E)$ to denote its approximate point spectrum. Since, as noted above,

$$
C_{N D}^{1}(X, \mathcal{T} X) \subset C_{N D}^{0}(X, \mathcal{T} X) \subset C(X, \mathcal{T} X)
$$

and since an $\epsilon$-eigenfunction for $T$ in $C_{N D}^{1}(X, \mathcal{T} X)$ is an $\epsilon$-eigenfunction for $T$ in $C_{N D}^{0}(X, \mathcal{T} X)$, one has

$$
\sigma_{\mathrm{ap}}\left(T ; C_{N D}^{1}(X, \mathcal{T} X)\right) \subset \sigma_{\mathrm{ap}}\left(T ; C_{N D}^{0}(X, \mathcal{T} X)\right) \subset \sigma_{\mathrm{ap}}(T ; C(X, \mathcal{T} X))
$$

Throughout the remainder of the paper the space $C_{N D}(X, \mathcal{T} X)$ may be taken to be either $C_{N D}^{0}(X, \mathcal{T} X)$ or $C_{N D}^{1}(X, \mathcal{T} X)$.

Theorem 2.1 (Spectral Mapping Theorem) In the space of divergencefree vector fields $C_{N D}(X, \mathcal{T} X)$ the spectrum $\sigma\left(M ; C_{N D}(X, \mathcal{T} X)\right)$ is invariant under vertical translations of the complex plane. Moreover, for each $t \neq 0$,

$$
\sigma\left(e^{t M}\right)=\exp t \sigma(M) .
$$


Since the proof of Theorem 2.1 is quite technical, we pause to discuss our strategy. Using standard facts from the theory of $C_{0}$-semigroups and by rescaling, we reduce the proof of Theorem 2.1 to the following main assertion (see our Lemma 2.7 below): If $1 \in \sigma_{\mathrm{ap}}(T), T:=T^{1}$ then $0 \in \sigma_{\mathrm{ap}}(M)$.

Our strategy for the proof of this main assertion develops some ideas of J. Mather [13]. The fact that $1 \in \sigma_{\mathrm{ap}}(T)$ implies the existence of an $\epsilon$ eigenfunction $u$ for $T$ for every $\epsilon>0$. That is, for every $\epsilon>0$, there is a vector field $u$, with unit norm such that $\|T u-u\| \leq \epsilon$. As in [13] and [21], the $\epsilon$-eigenfunctions of the operator $T$ have a nice feature: they can be "localized" along the trajectories of the flow. This means that for every $N \in \mathbb{N}$ there exist a point $x^{0} \in X$, a small neighborhood $D$ of this point, and a vector field $y$ with supp $y \subset \cup_{j=-N}^{N} \phi^{j}(D)$, such that $\|y-T y\|=O(1 / N)\|y\|$. In fact, starting from a given $\epsilon$-eigenfunction $u$ for $T$, define a "bump"-function $\alpha$ supported in $D$ and let $\gamma(j)=(N-|j|) / N$. The "localization" $y$ is defined to vanish outside $\cup_{j=-N}^{N} \phi^{j}(D)$ and by $y(x)=\gamma(j) \alpha\left(\phi^{-j} x\right)\left(T^{j} u\right)(x)$ for $x \in \phi^{j}(D)$ and $|j| \leq N$, equivalently,

$$
y(x)=\sum_{j=-N}^{N} \gamma(j)\left(T^{j} \alpha u\right)(x), \quad x \in X .
$$

To prove the main assertion above, our purpose is to construct a vector field $y$ with zero divergence such that $\|M y\|=O(1 / N)\|y\|$. We start with a divergence-free approximative eigenfunction $u$ of $T$. Since the set of divergence free vector fields is not closed under multiplication by "bump"functions, we can not use Mather's construction directly. Instead, we will construct a divergence free vector-field $w$, supported in a small neighborhood $D$ of a given point $x^{0} \in X$ in Lemma 2.3. The main part of this construction takes place in a special neighborhood $D$ of $x^{0}$, taken to be a thin and long "ellipsoid" with the longest axis directed along $u\left(x^{0}\right)$. The required vector field $w$ is constructed in the form $w(x)=\alpha(x) u\left(x^{0}\right)+w_{n}(x)$, where $\alpha$ is a "bump"- function, supported in $D$. The function $\alpha$ is chosen to have value identically one on a second thin and long "ellipsoid" $B$ contained in $D$. Some "fluid" leaks from the neighborhood $D$, but this can be recycled within a slightly larger neighborhood.

The desired almost-eigenfunction $y$ for $M$ is given by the formula

$$
y(x)=\int_{-\infty}^{\infty} \gamma(t) T^{t} w(x) d t, \quad x \in X
$$


where, as above, $\gamma$ vanishes outside of $[-N, N]$. Direct calculation shows that

$$
(M y)(x)=-\int_{-\infty}^{\infty} \gamma^{\prime}(t) T^{t} w(x) d t, \quad x \in X
$$

Since suppw $\subset D$, the support of the integrand in each integral belongs to $\left\{t \in \mathbb{R}:|t| \leq N\right.$ and $\left.\phi^{t} x \in D\right\}$. To obtain the desired inequality, we estimate $\|M y\|$ from above and $\|y\|$ from below. This requires some estimates of the sojourn time of the trajectory segment $\left\{\phi^{t}(x):|t| \leq N\right\}$ in $D$ and $B$. This is done in Lemma 2.5.

We start, for completeness, from the following simple lemma.

Lemma 2.2 If $x^{0} \in X$, then there is a coordinate chart at $x^{0}$, with coordinate functions $\left(x_{1}, \ldots, x_{n}\right)$, such that the local representation of the volume element on $X$ is just the usual volume $d x_{1} \wedge \cdots \wedge d x_{n}$ on $\mathbb{R}^{n}$. Moreover, if $z \in T_{x^{0}} X$, then the coordinates can be chosen so that the local representative of $z$ is $\|z\| \partial / \partial x_{2}$.

Proof: Let $y_{1}, \ldots, y_{n}$ denote local coordinates at $x^{0}$. Clearly, there is a nonvanishing density function $\rho: \mathbb{R}^{n} \rightarrow \mathbb{R}$ such that volume element is given by $\rho\left(y_{1}, \ldots, y_{n}\right) d y_{1} \wedge \cdots \wedge d y_{n}$. We seek new coordinates in the form

$$
y_{1}=f\left(x_{1}, \ldots, x_{n}\right), \quad y_{2}=x_{2}, \cdots, y_{n}=x_{n}
$$

where the volume element has the form

$$
\rho\left(f\left(x_{1}, \ldots, x_{n}\right), x_{2}, \ldots, x_{n}\right) \frac{\partial f}{\partial x_{1}}\left(x_{1}, \ldots, x_{n}\right) d x_{1} \wedge \cdots \wedge d x_{n} .
$$

There is a smooth function $f$, defined in a neighborhood of the origin in $\mathbb{R}^{n}$, such that

$$
\frac{\partial f}{\partial x_{1}}(0, \ldots, 0) \neq 0, \quad \frac{\partial f}{\partial x_{1}}\left(x_{1}, \ldots, x_{n}\right)=\left(\rho\left(f\left(x_{1}, \ldots, x_{n}\right), x_{2}, \ldots, x_{n}\right)\right)^{-1} .
$$

The first condition together with the Implicit Function Theorem implies the change of coordinates is invertible; the second condition ensures the volume element in the new coordinates has the desired form.

For the second statement of the lemma, note that the volume element is invariant under a rigid rotation of Euclidean space. 
A coordinate chart, as in the lemma, is called adapted to the volume on $X$ and the vector $z$. Of course, in the adapted coordinates, the Riemannian metric will not be the usual one, rather, it will have the form $\Sigma g_{i j}\left(x_{1}, \ldots, x_{n}\right) d x_{i} \otimes d x_{j}$ where the components $g_{i j}$ form a positive definite symmetric matrix of smooth functions. However, we make the following observation: if $u=\left(u_{1}, \ldots, u_{n}\right)$ is a vector field defined in an adapted coordinate chart, then

$$
\operatorname{div} u=\sum_{i=1}^{n} \frac{\partial u_{i}}{\partial x_{i}} .
$$

Suppose $z \in \mathcal{T}_{x^{0}} X$ is a tangent vector and let $\left(x_{1}, \ldots, x_{n}\right)$ denote local coordinates at $x^{0}$ adapted to the volume on $X$ and the vector $z$. If the adapted coordinate system is defined in a coordinate ball of diameter $\delta>0$ and if $a, b \in \mathbb{R}$ are such that $0<a<b<\delta / 8$, we define

$$
\begin{aligned}
D_{a, b}= & \left\{\left(x_{1}, \ldots, x_{n}\right):\left|x_{j}\right| \leq 4 b, j=1,2,\left|x_{j}\right| \leq a, j=3, \ldots, n\right\}, \\
B_{a, b}= & \left\{\left(x_{1}, \ldots, x_{n}\right):\left|x_{j}\right| \leq a / 2, j=1,3, \ldots, n,\left|x_{2}\right| \leq b / 2\right\} .
\end{aligned}
$$

Note that the closure of $B_{a, b}$ is contained in $D_{a, b}$. We say there is an $(a, b)$ divergence-free extension of the vector $z$ at $x^{0}$ if there is a smooth bumpfunction $\alpha: \mathbb{R}^{n} \rightarrow[0,1]$ with $\alpha(x)=1$ for $x \in B_{a, b}$ and $\alpha(x)=0$ for $x \notin D_{a, b}$ and a continuously differentiable vector field $w_{n}$ with support in $D_{a, b}$ such that

i) The vector field $w(x):=\alpha(x)\|z\| \partial / \partial x_{2}+w_{n}(x)$ is divergence free and has value $\|z\| \partial / \partial x_{2}$ in $B_{a, b}$,

ii) There is a number $C>0$ independent of $a, b$ such that $\left\|w_{n}\right\| \leq C a / b$.

Lemma 2.3 Every tangent vector on $X$ has an $(a, b)$ divergence-free extension.

Proof: Let $z \in \mathcal{T}_{x^{0}} X$. We will first prove the lemma for the case $n=2$. To construct a vector field $W$ with the required properties in the $\left(x_{1}, x_{2}\right)$ coordinate plane, consider the curves given by

$$
\frac{x_{1}^{4}}{a^{4}}+\frac{x_{2}^{4}}{b^{4}}=1, \quad \frac{x_{1}^{4}}{a^{4}}+\frac{x_{2}^{4}}{b^{4}}=2 .
$$


Let $\rho: \mathbb{R} \rightarrow[0,1]$ denote a smooth function such that $\rho(t)=1$ for $t \leq 1$, $\rho(t)=0$ for $t \geq 2$, and $\left|\rho^{\prime}(t)\right| \leq 3, t \in \mathbb{R}$. Also, define the sets

$$
\begin{aligned}
R & =\left\{\left(x_{1}, x_{2}\right):\left|x_{1}\right| \leq 2^{1 / 4} a,\left|x_{2}\right| \leq 2^{1 / 4} b\right\}, \\
S^{+} & =\left\{\left(x_{1}, x_{2}\right): \frac{\left(x_{1}-2^{1 / 4} a\right)^{4}}{a^{4}}+\frac{x_{2}^{4}}{b^{4}} \leq 2, x_{1} \geq 2^{1 / 4} a\right\}, \\
S^{-} & =\left\{\left(x_{1}, x_{2}\right): \frac{\left(x_{1}+2^{1 / 4} a\right)^{4}}{a^{4}}+\frac{x_{2}^{4}}{b^{4}} \leq 2, x_{1} \leq-2^{1 / 4} a\right\}
\end{aligned}
$$

and the functions $\theta: \mathbb{R}^{2} \rightarrow \mathbb{R}, f: \mathbb{R}^{2} \rightarrow \mathbb{R}$ and $\eta^{ \pm}: \mathbb{R} \rightarrow \mathbb{R}$ by

$$
\begin{aligned}
\theta\left(x_{1}, x_{2}\right) & =\rho\left(\frac{x^{4}}{a^{4}}+\frac{x_{2}^{4}}{b^{4}}\right), \\
f\left(x_{1}, x_{2}\right) & =-\frac{4 x_{2}^{3}\|z\|}{b^{4}} \int_{0}^{x_{1}} \rho^{\prime}\left(\frac{s^{4}}{a^{4}}+\frac{x_{2}^{4}}{b^{4}}\right) d s \\
\eta^{+}(\tau) & =-\frac{4\|z\|}{b^{4}} \int_{0}^{2^{1 / 4} a} \rho^{\prime}\left(\frac{s^{4}}{a^{4}}+\frac{\tau}{b^{4}}\right) d s \\
\eta^{-}(\tau) & =\frac{4\|z\|}{b^{4}} \int_{-2^{1 / 4} a}^{0} \rho^{\prime}\left(\frac{s^{4}}{a^{4}}+\frac{\tau}{b^{4}}\right) d s
\end{aligned}
$$

The vector field $w_{2}$ is defined in $R$ by $f\left(x_{1}, x_{2}\right) \partial / \partial x_{1}$, in $S^{+}$by

$$
\eta^{+}\left(\left(x_{1}-2^{1 / 4} a\right)^{4}+x_{2}^{4}\right) x_{2}^{3} \frac{\partial}{\partial x_{1}}-\eta^{+}\left(\left(x_{1}-2^{1 / 4} a\right)^{4}+x_{2}^{4}\right)\left(x_{1}-2^{1 / 4} a\right)^{3} \frac{\partial}{\partial x_{2}},
$$

in $S^{-}$by

$$
\eta^{-}\left(\left(x_{1}+2^{1 / 4} a\right)^{4}+x_{2}^{4}\right) x_{2}^{3} \frac{\partial}{\partial x_{1}}-\eta^{-}\left(\left(x_{1}+2^{1 / 4} a\right)^{4}+x_{2}^{4}\right)\left(x_{1}+2^{1 / 4} a\right)^{3} \frac{\partial}{\partial x_{2}}
$$

and $w_{2}$ is defined to vanish on the complement of $R \cup S^{+} \cup S^{-}$.

We will complete the proof for $n=2$ by showing the vector field

$$
W\left(x_{1}, x_{2}\right):=\theta\left(x_{1}, x_{2}\right)\|z\| \frac{\partial}{\partial x_{2}}+w_{2}\left(x_{1}, x_{2}\right)
$$

is the required extension of $z$.

A direct computation using (2.10) shows $\operatorname{div} W=0$ in the coordinate chart. Also, using the definition of $\rho$, we see the support of $W$ is in $D_{a, b}$. To 
show $W$ is $C^{1}$, just observe that $w_{2}$ and each of its first partial derivatives is continuous on the lines $x_{1}= \pm 2^{1 / 4} a$ and on the boundary of $R \cup S^{+} \cup S^{-}$. (We remark that additional smoothness can be obtained, if desired, by using the function $x_{1}^{k} / a^{k}+x_{2}^{k} / b^{k}$ with $k$ a sufficiently large positive integer in place of the choice $k=4$ used here.)

To obtain the required norm bound, let $G\left(x_{1}, \ldots, x_{n}\right)$ denote the matrix of the components $g_{i j}$ of the Riemannian metric in the adapted coordinates. The square of the norm of a vector $V$ at $x=\left(x_{1}, \ldots, x_{n}\right)$ is then given by $\langle G(x) V, V\rangle$. Thus, if $\|G\|$ denotes the supremum of the matrix norms over the points in the chart, we have $\|V\| \leq\|G\||V|$ where the single bars denote the usual norm in $\mathbb{R}^{n}$. Then, for example, using the usual estimate for the integral in the definition of $f$, we estimate the norm of $w_{2}$ in $R$ by

$$
\|G\| \sup \left|f\left(x_{1}, x_{2}\right)\right| \leq\|G\|\left(4\|z\| 2^{3 / 4} b^{3} / b^{4}\right) 3\left(2^{1 / 4} a\right) \leq C_{1} a / b
$$

where the constant $C_{1}$ does not depend on $a$ or $b$. Similarly, we can estimate the norm of $w_{2}$ in $S^{ \pm}$. For example, in $S^{+}$we find the upper bound

$$
\|G\| \sup \left|\eta^{+}\left(\left(x_{1}-2^{1 / 4} a\right)^{4}+x_{2}^{4}\right)\right|\left(\left(2^{1 / 4} b\right)^{6}+\left(2^{1 / 4} a\right)^{6}\right)^{1 / 2} \leq C_{2} a / b .
$$

To prove the lemma for the case $n \geq 3$, we will show how to extend the vector field $W$ defined above to a vector field on $\mathbb{R}^{n}$ with the required properties. To do this, let $\chi: \mathbb{R} \rightarrow[0,1]$ denote a smooth "bump"-function such that $\chi(t)=1$ for $|t| \leq \frac{a}{2}$ and $\chi(t)=0$ for $|t| \geq a$, and define

$$
\Psi\left(x_{3}, \ldots, x_{n}\right)=\prod_{j=3}^{n} \chi\left(x_{j}\right) .
$$

The required vector field $w$ is given by

$$
w\left(x_{1}, \ldots, x_{n}\right)=\Psi\left(x_{3}, \ldots, x_{n}\right) W\left(x_{1}, x_{2}\right) .
$$

The fact that the new vector field $w$ is continuously differentiable, agrees with $\|z\| \partial / \partial x_{2}$ in $B_{a, b}$, and is supported in $D_{a, b}$ is clear. Also, since the range of $\Psi$ is the unit interval, the norm bound on $w_{n}$ is the same as the norm bound on $w_{2}$. To complete the proof we must show $\operatorname{div} w=0$. But, since the vector field $w$ has nonzero components only in the first two coordinate directions,

$$
\operatorname{div} w\left(x_{1}, \ldots, x_{n}\right)=\Psi\left(x_{3}, \ldots, x_{n}\right) \operatorname{div} W\left(x_{1}, x_{2}\right)=0
$$


as required.

To estimate the time that trajectories spend in a neighborhood of $x^{0}$, we first need the following observation.

Lemma 2.4 Suppose $x^{0} \in X$ is not a periodic point for the vector field $v$ with flow $\phi^{t}$. If $N$ is a positive integer and $0<s \leq N$, then there is a $\delta>0$ such that every neighborhood $D$ containing $x^{0}$, with $\operatorname{diam} D \leq \delta$, has the following property: if $x \in D$, then $\phi^{t} x \notin D$ for $s \leq|t| \leq N$.

Proof: Suppose the lemma is false and, for each positive integer $k$, let $D_{k}$ denote the ball centered at $x^{0}$ with radius $1 / k$. For each $k$, there is some $x_{k} \in D_{k}$ and some $t_{k}$ in the set $J:=\{t: 0<s \leq|t| \leq N\}$ with $\phi^{t_{k}}\left(x_{k}\right)$ in $D_{k}$. Since $J \times \bar{D}_{1}$ for the closure $\bar{D}_{1}$ of $D_{1}$ is compact, there is a convergent subsequence of the pairs $\left(t_{k}, x_{k}\right)$. But, by the choice of $D_{k}$, the second component of this sequence converges to $x^{0}$ and, by the compactness of $J$, the limit $T$ of the first component satisfies $|T| \geq s>0$. The continuity of the flow ensures that $\phi^{T}\left(x^{0}\right)=x^{0}$, in contradiction to the fact that $x^{0}$ is not periodic.

Consider a vector field $v$ on $X$ tangent to the flow $\phi^{t}$. For each open set $U \subset X$, each non negative integer $N$ and each point $x \in X$, define

$$
\begin{aligned}
\Theta_{N, U}(x) & :=\left\{t \in \mathbb{R}:|t| \leq N, \phi^{t} x \in U\right\} \\
m_{N, U}(x) & :=\operatorname{mes}\left(\Theta_{N, U}(x)\right) .
\end{aligned}
$$

Lemma 2.5 Suppose $X$ has dimension $n \geq 3$. If $\epsilon>0$, then there is a constant $K>0$ such that for each non periodic point $x^{0}$ and positive integer $N$ there is a pair of numbers $a, b$ such that $\epsilon>b>a>0$ and $a / b \leq \epsilon$ together with a pair of open sets $B, D$ at $x^{0}$ such that $B \subset B_{a, b}$ and $D_{a, b} \subset D$, with the following property: for each $x \in X$,

$$
\frac{m_{N, D}(x)}{m_{N, B}\left(x^{0}\right)} \leq K
$$

Proof: By Lemma 2.4, there is a neighborhood $\widehat{D}$ at $x^{0}$ such that $\phi^{t} y \notin \widehat{D}$ whenever $y \in \widehat{D}$ and $1 / 2 \leq|t| \leq 2 N$. Suppose $K, a, b, B, D$, with $D \subseteq \widehat{D}$, are given so that the inequality (2.12) holds for $x \in D$. We claim that 
(2.12) holds for all $x \in X$. To see this, note first that, for $y \in D$, we have $\Theta_{N, D}(y)=\Theta_{2 N, D}(y)$. Thus, by our definition, $m_{N, D}(y)=m_{2 N, D}(y)$. If $x \in X$ and $D \cap\left\{\phi^{t} x:|t| \leq N\right\}=\emptyset$, then $m_{N, D}(x)=0$ and (2.12) holds. Otherwise, fix $y \in D \cap\left\{\phi^{t} x:|t| \leq N\right\}$. Since $\Theta_{N, D}(x) \subset \Theta_{2 N, D}(y)$, we have

$$
m_{N, D}(x) \leq \max _{y \in D} m_{2 N, D}(y)=\max _{y \in D} m_{N, D}(y) \leq K \cdot m_{N, D}\left(x^{0}\right),
$$

as required.

To complete the proof, we will construct $K, a, b, B, D$ so that (2.12) holds for $x \in D$. This will require several steps.

Step 1. We will work in an adapted coordinate system at $x^{0}$ with coordinate functions $\left(x_{1}, \ldots, x_{n}\right)$. We will determine the required sets $B, D$ for appropriate $a, b$ in the form

$$
\begin{aligned}
& B=\left\{x: \frac{x_{1}^{4}}{(a / 2)^{4}}+\frac{x_{2}^{4}}{(b / 2)^{4}}+\sum_{j=3}^{4} \frac{x_{j}^{4}}{(a / 2)^{4}} \leq 1\right\}, \\
& D=\left\{x: \frac{x_{1}^{4}}{(4 n b)^{4}}+\frac{x_{2}^{4}}{(4 n b)^{4}}+\sum_{j=3}^{4} \frac{x_{j}^{4}}{(n a)^{4}} \leq 1\right\} .
\end{aligned}
$$

If $a<b$, then, clearly, $B \subset B_{a, b}$ and $D_{a, b} \subset D$. We will show that there is a constant $K$ such that for some choice of $a, b$ and $a / b$ all sufficiently small, the inequality (2.12) is valid for the corresponding set $D$.

We will use the following auxiliary constructions:

For each $\delta>0$, let $S_{\delta}$ denote a section for $v$ at the origin of the coordinate system, that is, at $x^{0}$, such that the Riemannian diameter $\operatorname{diam} S_{\delta}<\delta$, and define $\Sigma_{\delta}:=\left\{\phi^{t} \sigma: \sigma \in S_{\delta},|t| \leq \delta\right\}$. Consider the local representation of $v$ given by $\sum_{i=1}^{n} v_{i}(x) \partial / \partial x_{i}$. By a rigid rotation, if necessary, we can and will arrange the adapted coordinates so that $v_{1}(0)=0$. Also, we define $V$ by

$$
V(x):=\sum_{i=3}^{n} v_{i}^{4}(x), \quad x \in \Sigma_{\delta}
$$

and the number

$$
M_{\delta}:=\max \left\{\max _{x \in \Sigma_{\delta}}\left|v_{1}(x)\right|, \quad \max _{x \in \Sigma_{\delta}}\left(\sum_{i=3}^{n} v_{i}^{4}(x)\right)^{1 / 4}\right\} .
$$


If $V\left(x^{0}\right)=0$, then $\lim _{\delta \rightarrow 0} M_{\delta} \rightarrow 0$. In case $V\left(x^{0}\right)=0$ and $M_{\delta} \neq 0$ for every $\delta$, we will only consider $a, b$ such that

$$
a=b \cdot\left(M_{\delta}\right)^{1 / 2} .
$$

Of course, even under the restriction just imposed, $a, b, a / b$ can each be chosen arbitrary small. If $V\left(x^{0}\right) \neq 0$ or if $V\left(x^{0}\right)=0$ and $M_{\delta} \equiv 0$ for all sufficiently small $\delta$, we ignore this restriction.

Step 2. For each $\delta$, Lemma 2.4 and the definition of $\Sigma_{\delta}$ together imply there is an open ball $A_{\delta} \subset \Sigma_{\delta}$ at the origin such that, for each $x \in A_{\delta}$ and for each time $t$ with $|t|>\delta$, the point $\phi^{t} x$ is not in $\Sigma_{\delta}$. If $\delta>0$ is given, choose $a, b$ as required in (2.13) and so small that $D$ is in $A_{\delta}$. If $x \in D$, let $x^{\prime}$ denote the point on $\partial D$ where the segment of the trajectory $\left\{\phi^{t} x:|t| \leq N\right\}$ first enters $D$ and let $x^{\prime \prime}=\phi^{t_{D}} x^{\prime}$ denote the point of $\partial D$ where the segment of the trajectory $\left\{\phi^{t} x:|t| \leq N\right\}$ last exits $D$. Clearly, $m_{N, D}(x) \leq t_{D}$.

We use the Mean Value Theorem for integrals on the $j$ th component of the vector field $v$ to obtain a point $\xi^{j} \in \Sigma_{\delta}$ such that

$$
x_{j}^{\prime \prime}=x_{j}^{\prime}+\int_{0}^{t_{D}} v_{j}\left(\phi^{t} x\right) d t=x_{j}^{\prime}+t_{D} v_{j}\left(\xi^{j}\right) .
$$

For each $j=1, \ldots, n$, let $v_{j}^{*}=v_{j}\left(\xi^{j}\right)$. Also, as an abbreviation, define $\alpha_{j}=4 n b$ for $j=1,2$ and $\alpha_{j}=n a$ for $j=3, \ldots, n$.

Since $x^{\prime}$ and $x^{\prime \prime}$ both belong to $\partial D$, we have

$$
\sum_{i=1}^{n}\left(\frac{x_{i}^{\prime}}{\alpha_{i}}\right)^{4}=1 \quad \text { and } \quad \sum_{i=1}^{n}\left(\frac{x_{i}^{\prime}+t_{D} v_{i}^{*}}{\alpha_{i}}\right)^{4}=1 .
$$

Using a standard inequality for the norm $\left\|\left(\gamma_{i}\right)\right\|=\left(\sum_{i}\left|\gamma_{i}\right|^{4}\right)^{1 / 4}$, we find

$$
\begin{gathered}
1=\sum_{i=1}^{n}\left(\frac{x_{i}^{\prime}+t_{D} v_{i}^{*}}{\alpha_{i}}\right)^{4} \geq\left(\sum_{i=1}^{n}\left(\frac{t_{D} v_{i}^{*}}{\alpha_{i}}\right)^{4}\right)^{1 / 4}-\left(\sum_{i=1}^{n}\left(\frac{x_{i}^{\prime}}{\alpha_{i}}\right)^{4}\right)^{1 / 4} \\
=\left(\sum_{i=1}^{n}\left(\frac{t_{D} v_{i}^{*}}{\alpha_{i}}\right)^{4}\right)^{1 / 4}-1 .
\end{gathered}
$$

This computation yields the estimate

$$
t_{D}^{4} \sum_{i=1}^{n}\left(\frac{v_{i}^{*}}{\alpha_{i}}\right)^{4} \leq 2^{4}
$$


Step 3. Consider the time $t_{B}$ when the segment of the trajectory $\left\{\phi^{t} x^{0}\right.$ : $|t| \leq N\}$ first leaves $D$. Clearly, $m_{N, B}\left(x^{0}\right) \geq t_{B}$.

Recall that in our local coordinates, $x^{0}$ resides at the origin and define $\tilde{x}=\phi^{t_{B}}\left(x^{0}\right)$. As in Step 2, by the the Mean Value Theorem, there is some $\eta^{j} \in \Sigma_{\delta}$ such that

$$
\tilde{x}_{j}=\int_{0}^{t_{B}} v_{j}\left(\phi^{t}\left(x^{0}\right)\right) d t=t_{B} v_{j}\left(\eta^{j}\right) .
$$

Define $\tilde{v}_{j}:=v_{j}\left(\eta^{j}\right)$, the numbers $\beta_{j}=a / 2$ for $j=1,3, \ldots, n$ and $\beta_{2}=b / 2$. Since $\tilde{x} \in \partial B$, we have

$$
t_{B}^{4} \sum_{j=1}^{n}\left(\frac{\tilde{v}_{j}}{\beta_{j}}\right)^{4}=1 .
$$

Step 4. In accordance with the previous notation, we define

$$
\tilde{V}=\sum_{i=3}^{n} \tilde{v}_{i}^{4}, \quad V^{*}=\sum_{i=3}^{n}\left(v_{i}^{*}\right)^{4} .
$$

We use (2.14)-(2.15) to obtain the estimate $\left(t_{D} / t_{B}\right)^{4} \leq 2^{16} n^{4} \cdot d$ where

$$
d:=\frac{\tilde{v}_{1}^{4}+\left(\frac{a}{b} \tilde{v}_{2}\right)^{4}+\tilde{V}}{\left(\frac{a}{b} v_{1}^{*}\right)^{4}+\left(\frac{a}{b} v_{2}^{*}\right)^{4}+4^{4} V^{*}} .
$$

We will show that for all sufficiently small $\delta>0$, there are some choices of $a, b$ such that $d<2$. There are several cases.

Case 1. If $V\left(x^{0}\right) \neq 0$, then $\lim _{\delta \rightarrow 0} d=4^{-4}$.

Case 2. If $V\left(x^{0}\right)=0$ and $M_{\delta} \equiv 0$ for all sufficiently small $\delta$, then, since $v_{2}\left(x^{0}\right) \neq 0, \lim _{\delta \rightarrow 0} d=1$.

Case 3. Suppose $V\left(x^{0}\right)=0$ and $M_{\delta} \neq 0$. However, note that we still have $\lim _{\delta \rightarrow 0} M_{\delta} \rightarrow 0$. Also, the restriction we imposed in (2.13) provides that

In this case, we have

$$
\frac{b}{a} \tilde{v}_{1}^{1 / 2} \leq 1, \quad \frac{b}{a} \tilde{V}^{1 / 8} \leq 1
$$

$$
d=\frac{\left(\frac{b}{a} \tilde{v}_{1}\right)^{4}+\tilde{v}_{2}^{4}+\left(\frac{b}{a}\right)^{4} \tilde{V}}{\left(v_{1}^{*}\right)^{4}+\left(v_{2}^{*}\right)^{4}+4^{4}\left(\frac{b}{a}\right)^{4} V^{*}} \leq \frac{\tilde{v}_{1}^{2}+\tilde{v}_{2}^{4}+\tilde{V}^{1 / 2}}{\left(v_{2}^{*}\right)^{4}} .
$$


Passing to the limit as $\delta \rightarrow 0$, we see that the last expression converges to 1.

We need the following elementary fact.

Lemma 2.6 Suppose $A$ denotes an invertible bounded operator on a Banach space $E$ and let $N \in \mathbb{Z}$. If $N \geq 2$ and $1 \in \sigma_{a p}(A)$, then there is a vector $u \in E$ with $\|u\|_{E}=1$ such that $\left\|A^{k} u\right\|_{E} \leq 2$ for each integer $k$ with $|k| \leq N$.

Proof: Set $\epsilon=\left(\sum_{k=-N}^{N}\left\|A^{k}\right\|\right)^{-1}$. Since $1 \in \sigma_{\mathrm{ap}}(A)$, there is some $u \in E$ with $\|u\|_{E}=1$ such that $\|A u-u\| \leq \epsilon$. Also, for $1 \leq|k| \leq N$, note that

$A^{k}-I=\left(\sum_{j=0}^{k-1} A^{j}\right)(A-I), k>0, \quad A^{k}-I=-\left(\sum_{j=-1}^{k} A^{j}\right)(A-I), k<0$.

Hence, for $|k| \leq N$, we have $\left\|A^{k} u-u\right\| \leq \sum_{j=-N}^{N}\left\|A^{j}\right\| \cdot\|A u-u\| \leq 1$ and, as a result, $\left\|A^{k} u\right\| \leq\left\|A^{k} u-u\right\|+\|u\| \leq 2$.

The main result of this section is the following lemma.

Lemma 2.7 Suppose $e^{t M}=T^{t}$ is the group defined in (1.5) and define $T:=$ $T^{1}$. If $1 \in \sigma_{a p}(T ; C(X, \mathcal{T} X))$, then $\sigma_{a p}\left(M ; C_{N D}(X, \mathcal{T} X)\right)$ contains the imaginary axis of the complex plane.

In accordance to $(2.8)$ this lemma also shows that $1 \in \sigma_{\mathrm{ap}}\left(T, C_{N D}(X, \mathcal{T} X)\right)$ implies $0 \in \sigma_{\mathrm{ap}}\left(M, C_{N D}(X, \mathcal{T} X)\right)$ for both cases:

$$
C_{N D}(X, \mathcal{T} X)=C_{N D}^{0}(X, \mathcal{T} X) \text { or } C_{N D}(X, \mathcal{T} X)=C_{N D}^{1}(X, \mathcal{T} X) .
$$

Proof: Let $\xi \in \mathbb{R}$ and let $K$ be defined as in Lemma 2.4. Also, for notational convenience, define $\omega:=1 /(12 K)$.

Since $1 \in \sigma_{\mathrm{ap}}(T)$, Lemma 2.6 applied to the bounded linear operator $T=T^{1}$ ensures that, for each integer $N \geq 2$, there is some vector field $u \in C(X, \mathcal{T} X)$ such that

$$
\begin{array}{r}
\|u\|_{C(X, \mathcal{T} X)}=1 \\
\left\|T^{k} u\right\|_{C(X, \mathcal{T} X)} \leq 2 \text { for }|k| \leq N .
\end{array}
$$


Since $\left\{T^{t}\right\}$ is a $C_{0}$-semigroup, $T^{t} u \rightarrow u$ in $C(X, \mathcal{T} X)$ as $t \rightarrow 0$. Thus, there is a real number $s$ with $0<s \leq 2 N$ such that

$$
\begin{aligned}
\left\|T^{t} u-u\right\|_{C(X, \mathcal{T} X)} & \leq \omega \text { for }|t| \leq s, \\
\left|e^{-i \xi t}-1\right| & \leq \omega \text { for }|t| \leq s .
\end{aligned}
$$

Also, there is a smooth function $\gamma: \mathbb{R} \rightarrow[0,1]$ such that:

$$
\begin{array}{r}
\gamma(t)=0 \text { for }|t| \geq N, \\
\left|\gamma^{\prime}(t)\right| \leq \frac{2}{N}, \text { for } t \in \mathbb{R}, \\
\gamma(t)=1 \text { for }|t| \leq s .
\end{array}
$$

In view of (2.16), and the fact that the non periodic points are dense in $X$, there is a non periodic point $x^{0} \in X$ such that

$$
\left\|u\left(x^{0}\right)\right\| \geq \frac{1}{2}\|u\|_{C(X, \mathcal{T} X)}=\frac{1}{2} .
$$

Use Lemma 2.5 to find small $a, b$ with small $a / b$, and neighborhoods $D \supset B \ni$ $x^{0}$, such that (2.12) holds. Moreover, in accordance with Lemma 2.4, we can choose $a, b$ sufficiently small so that for $D:=D_{a, b}$, for $s$ from (2.18)-(2.19) and with $c:=\max _{|t| \leq 1}\left\|T^{t}\right\|$ we have

$$
\phi^{t} y \notin D \text { for any } y \in D \text { provided } s \leq|t| \leq 2 N
$$

and, for some constant $C$, the following inequalities:

$$
\begin{aligned}
C \frac{a}{b}\left(\max _{|t| \leq N}\left\|T^{t}\right\|\right) & \leq \omega, \\
\max _{y \in D,|t| \leq N}\left\|\Phi(y, t) u\left(x^{0}\right)\right\| & \leq 4 c .
\end{aligned}
$$

For the last inequality, we use (2.17) to show that

$$
\begin{aligned}
& \max _{|t| \leq N}\left\|\Phi\left(x^{0}, t\right) u\left(x^{0}\right)\right\| \leq \max _{|t| \leq N, x \in X}\|\Phi(x, t) u(x)\| \\
& \leq \max _{|t| \leq N, x \in X}\left\|\Phi\left(\phi^{-t} x, t\right) u\left(\phi^{-t} x\right)\right\|=\max _{|k| \leq N-1,|\tau| \leq 1}\left\|T^{k+\tau} u\right\| \\
& \leq c \max _{|k| \leq N-1}\left\|T^{k}\right\| \leq 2 c .
\end{aligned}
$$


Since $\Phi:(x, t) \mapsto \Phi(x, t)$ is uniformly continuous on the compact set $X \times$ $[-N, N]$, we have $(2.26)$ for a sufficiently small neighborhood $D$ of $x^{0}$.

We use Lemma 2.3 with $z=u\left(x^{0}\right)$. After a rigid rotation, if necessary, we can arrange the adapted coordinates so that the component of $v\left(x^{0}\right)$ in the direction of the first coordinate vanishes. Then, for this choice of adapted coordinates, there is a divergence-free vector field of the form

$$
w(x)=\alpha(x) u\left(x^{0}\right)+w_{n}(x)
$$

with $\alpha$ and $w_{n}$ supported in $D$,

$$
\left\|w_{n}\right\|_{C(X, \mathcal{T} X)} \leq C \frac{a}{b}
$$

and $\alpha(x)=1$ for $x \in B_{a, b}$.

Define the vector field $y$ on $X$ by

$$
y(x)=\int_{-\infty}^{\infty} e^{-i \xi t} \gamma(t) T^{t} w(x) d t
$$

We see that $y$ has zero divergence (for this remember $T^{t}$ preserves the divergence-free vector fields). By easy computations with $M y=\left.\frac{d}{d \tau} T^{\tau} y\right|_{\tau=0}$ one has:

$$
M y=\left.\int_{-\infty}^{\infty} \frac{d}{d \tau}\left(e^{-i \xi(t-\tau)} \gamma(t-\tau)\right)\right|_{\tau=0} T^{t} w d t=i \xi y-\int_{-\infty}^{\infty} \gamma^{\prime}(t) e^{-i \xi t} T^{t} w d t
$$

To complete the proof, we must show that $i \xi \in \sigma_{a p}\left(M ; C_{N D}(X, \mathcal{T} X)\right)$. This is an immediate consequence of the following proposition: There is a number $A>0$ that does not depend on the choice of $N$ such that

$$
\left\|\int_{-\infty}^{\infty} \gamma^{\prime}(t) e^{-i \xi t} T^{t} w d t\right\|_{C(X, \mathcal{T} X)} \leq \frac{A}{N}\|y\|_{C(X, \mathcal{T} X)} .
$$

To prove the proposition, fix $x \in X$ and note that

$$
\|M y(x)-i \xi y(x)\|=\left\|\int_{-\infty}^{\infty} e^{-i \xi t} \gamma^{\prime}(t)\left(T^{t} w\right)(x) d t\right\| \leq I_{1}+I_{2}
$$

where, by (2.20) and (2.21),

$$
\begin{aligned}
& I_{1}=\frac{2}{N} \int_{-N}^{N} \alpha\left(\phi^{-t} x\right)\left\|\Phi^{t}\left(\phi^{-t} x, t\right) u\left(x^{0}\right)\right\| d t, \\
& I_{2}=\frac{2}{N} \int_{-N}^{N}\left\|\Phi\left(\phi^{-t} x, t\right)\right\|\left\|w_{n}\left(\phi^{-t} x\right)\right\| d t .
\end{aligned}
$$


Since supp $\alpha \subset D$ and $\operatorname{supp} w_{n} \subset D$, the integrations $I_{1}$ and $I_{2}$ can be restricted to $\Theta_{D}(x)=\Theta_{N, D}(x)$, see the notation in (2.11). We use (2.26) to obtain:

$$
I_{1} \leq \frac{2}{N} \int_{\Theta_{D}(x)} \max _{y \in D,|t| \leq N}\left\|\Phi(y, t) u\left(x^{0}\right)\right\| \leq \frac{2}{N} 4 c \cdot m_{N, D}(x) .
$$

We use (2.25) and (2.28) to estimate $I_{2}$ :

$$
\begin{aligned}
I_{2} & \leq \frac{2}{N} \int_{\Theta_{D}(x)} \max _{y \in D,|y| \leq N}\|\Phi(y, t)\|\left\|w_{n}\right\|_{C(X, \mathcal{T} X)} d t \\
& \leq \frac{2}{N} \max _{|t| \leq N}\left\|T^{t}\right\| \cdot C \frac{a}{b} \cdot m_{N, D}(x) \leq \frac{2}{N} \omega m_{N, D}(x) .
\end{aligned}
$$

We obtain the desired upper estimate from (2.29) and (2.30), namely,

$$
\|M y-i \xi y\|_{C(X, \mathcal{T} X)} \leq \frac{A_{1}}{N} \max _{x \in X} m_{N, D}(x) .
$$

To determine the lower bound, we define

$$
\begin{aligned}
& J_{1}=\left\|\int_{-\infty}^{\infty} e^{-i \xi t} \gamma(t) \alpha\left(\phi^{-t} x^{0}\right) u\left(x^{0}\right) d t\right\|, \\
& J_{2}=\left\|\int_{-\infty}^{\infty} e^{-i \xi t} \gamma(t) \alpha\left(\phi^{-t} x^{0}\right)\left[\left(T^{t} u\right)\left(x^{0}\right)-u\left(x^{0}\right)\right] d t\right\|, \\
& J_{3}=\left\|\int_{-\infty}^{\infty} e^{-i \xi t} \gamma(t)\left(T^{t} w_{n}\right)\left(x_{0}\right)\right\|
\end{aligned}
$$

and note that

$$
\|y\|_{C(X, \mathcal{T} X)} \geq\left\|y\left(x_{0}\right)\right\| \geq J_{1}-J_{2}-J_{3} .
$$

Again, each integral is equal to its restriction to $\Theta_{D}\left(x^{0}\right)=\Theta_{N, D}\left(x^{0}\right)$.

As in (2.30), we use (2.28) and (2.25) to estimate $J_{3}$ from above:

$$
J_{3} \leq \int_{\Theta_{D}\left(x^{0}\right)}\left\|\left(T^{t} w_{n}\right)\left(x^{0}\right)\right\| d t \leq \omega m_{N, D}\left(x^{0}\right) .
$$

Next, we use (2.18) to estimate $J_{2}$ from above. For this, note that from (2.24) if $t \in \Theta_{D}\left(x^{0}\right)$, then $|t|<s$. Thus, we have

$$
J_{2} \leq \int_{\Theta_{D}\left(x^{0}\right)}\left\|T^{t} u-u\right\|_{C(X, \mathcal{T} X)} d t \leq \omega m_{N, D}\left(x^{0}\right) .
$$


Finally, we estimate $J_{1}$ from below:

$$
J_{1}=\left\|u\left(x^{0}\right)\right\|\left|\int_{\Theta_{D}\left(x^{0}\right)} e^{-i \xi t} \gamma(t) \alpha\left(\phi^{-t} x^{0}\right) d t\right| \geq J_{11}-J_{12},
$$

where

$$
\begin{aligned}
& J_{11}=\left|\int_{\Theta_{D}\left(x^{0}\right)} \gamma(t) \alpha\left(\phi^{-t} x^{0}\right) d t\right| \cdot\left\|u\left(x^{0}\right)\right\| \\
& J_{12}=\left|\int_{\Theta_{D}\left(x^{0}\right)}\left(e^{-i \xi t}-1\right) \gamma(t) \alpha\left(\phi^{-t} x^{0}\right) d t\right| \cdot\left\|u\left(x^{0}\right)\right\| .
\end{aligned}
$$

Since, by (2.24), $\phi^{t} x^{0} \notin D$ for $s \leq|t| \leq 2 N$, equation (2.22) gives $\gamma(t)=1$ for $t \in \Theta_{D}\left(x^{0}\right)$. As $\alpha(x)=1$ for $x \in B$, we use (2.23) to compute the estimate:

$$
J_{11} \geq \frac{1}{2} \int_{\Theta_{B}\left(x^{0}\right)} \alpha\left(\phi^{-t} x^{0}\right) d t \geq \frac{1}{2} m_{N, B}\left(x^{0}\right) .
$$

Since $\left\|u\left(x^{0}\right)\right\| \leq 1$ and $|t| \leq s$ for $t \in \Theta_{D}\left(x^{0}\right)$, the inequality (2.19) implies:

$$
J_{12} \leq \int_{\Theta_{D}\left(x^{0}\right)}\left|e^{-i \xi t}-1\right| \alpha\left(\phi^{-t} x^{0}\right) d t \leq \omega m_{N, D}\left(x^{0}\right) .
$$

The estimates $(2.35),(2.36),(2.33),(2.32)$, and (2.12) together with the our choice of $\omega=1 /(12 K)$ give the following:

$$
\begin{aligned}
\|y\|_{C(X, \mathcal{T} X)} & \geq \frac{1}{2} m_{N, B}\left(x^{0}\right)-3 \omega m_{N, D}\left(x^{0}\right) \\
& \geq \frac{1}{2 K} \max _{x \in X} m_{N, D}(x)-3 \omega \max _{x \in X} m_{N, D}(x) \\
& =\frac{1}{4 K} \max _{x \in X} m_{N, D}(x) .
\end{aligned}
$$

By combining this estimate with (2.31), we have the desired result.

We are now in the position to prove Theorem 2.1.

Proof: It is well-known (see, e.g., [19]) that the Spectral Inclusion Theorem

$$
\sigma\left(e^{t M}\right) \supset \exp t \sigma(M), \quad t \neq 0
$$


holds for any $C_{0}$-semigroup. Also, the spectral mapping theorem is true for the point and residual spectrum. Therefore, to prove (2.9) one needs to show that, in $C_{N D}(X, \mathcal{T} X)$,

$$
\sigma_{\operatorname{ap}}\left(e^{t M}\right) \subset \exp t \sigma_{\operatorname{ap}}(M), \quad t \neq 0 .
$$

Fix $\mu=|\mu| e^{i \theta} \in \sigma_{\operatorname{ap}}\left(e^{t M} ; C_{N D}(X, \mathcal{T} X)\right)$. Then $\mu=e^{t \lambda}$ for $\lambda=\frac{1}{t} \ln |\mu|+$ $i \frac{\theta}{t}$. Consider the cocycle $\tilde{\Phi}(x, t)=e^{-t \lambda} \Phi(x, t)$, and the group $\left\{\tilde{T}^{t}\right\}, \tilde{T}^{t}=$ $e^{t \tilde{M}}$, defined by $\tilde{\Phi}(x, t)$ as in (1.5). Then $\mu \in \sigma_{\mathrm{ap}}\left(e^{t M}, C_{N D}(X, \mathcal{T} X)\right)$ implies that $1 \in \sigma_{\mathrm{ap}}\left(e^{t \tilde{M}} ; C_{N D}(X, \mathcal{T} X)\right)$. By (2.8) and Lemma 2.7, we have $0 \in \sigma_{\mathrm{ap}}\left(\tilde{M} ; C_{N D}(X, \mathcal{T} X)\right)$. But, since $\tilde{M}=M-\lambda$, this implies $\lambda \in$ $\sigma_{\mathrm{ap}}\left(M ; C_{N D}(X, \mathcal{T} X)\right)$.

To prove that $\sigma(M)$ is invariant under the translations along the imaginary axis, we fix $\lambda \in \sigma_{\mathrm{ap}}(M)$ and $\xi \in \mathbb{R}$. By the Spectral Inclusion Theorem for $t=1$ we have $1 \in \sigma_{\text {ap }}\left(e^{\tilde{M}}\right)$, also, $\tilde{M}=M-\lambda$. By Lemma 2.7, one has $i \xi \in \sigma_{\mathrm{ap}}(\tilde{M})$ and, as a result, $\lambda+i \xi \in \sigma_{\mathrm{ap}}(M)$.

We will use notations

$$
s(A):=\sup \{\operatorname{Re} z: z \in \sigma(A)\} \text { and } \omega(A):=\lim _{t \rightarrow \infty} t^{-1} \ln \left\|e^{t A}\right\|
$$

for the spectral bound of a generator $A$ and the growth bound of a $C_{0^{-}}$ semigroup $\left\{e^{t A}\right\}$, respectively. Note [19], that for an arbitrary $C_{0}$-semigroup $\left\{e^{t A}\right\}$ one has $s(A) \leq \omega(A)$, but, generally, $s(A) \neq \omega(A)$. The Spectral Mapping Theorem, however, gives for the group of weighted composition operators the following fact.

Corollary 2.8 In the space of continuous divergence free vector fields the spectral bound and the growth bound are equal: $s(M)=\omega(M)$.

Remark 2.9. Consider the kinematic dynamo operator $L_{\varepsilon}=L+\varepsilon \Delta$ with $\varepsilon>0$ (see (1.1)). This is an elliptic operator, it generates an analytic semigroup, and the spectral mapping theorem is valid [19] for this semigroup. Hence, $s\left(L_{\varepsilon}\right)=\omega\left(L_{\varepsilon}\right)$ for $\varepsilon>0$. For $L_{0}=L$, Corollary 2.8 shows that this equality is also valid for $\varepsilon=0$.

Remark 2.10. M. Vishik [23] has shown that $\lim _{\sup _{\varepsilon \rightarrow 0}} \omega\left(L_{\varepsilon}\right) \leq \omega\left(L_{0}\right)$. In view of Remark 2.9, this theorem can be reformulated as $\lim _{\sup _{\varepsilon \rightarrow 0}} s\left(L_{\varepsilon}\right) \leq$ 
$s\left(L_{0}\right)$. We stress that the last assertion does not involve the construction of the group $\left\{e^{t L_{\varepsilon}}\right\}$; it is given in the terms of generators only. See Remark 3.9 below for the connection of this assertion to the "fast"-dynamo problem. Our formulation suggests that the validity of the assertion $\lim _{\sup _{\varepsilon \rightarrow 0}} s\left(L_{\varepsilon}\right)=$ $s\left(L_{0}\right)$ can be approached as a problem from the theory of singular perturbations for the generators of $C_{0}$-semigroups.

Remark 2.11. The Spectral Mapping Theorem for semigroups of weighted composition operators does not hold without the assumption that aperiodic trajectories are dense in $X$ (see $[5,12]$ for examples). However, in the space $C(X, \mathcal{T} X)$ (and $L_{2}$, see $[5,12]$ ), this assumption is not required to prove the following Annular Hull Theorem:

$$
\exp t \sigma(M) \subset \sigma\left(e^{t M}\right) \subset \mathcal{H}(\exp t \sigma(M)), \quad t \neq 0,
$$

where $\mathcal{H}(\cdot)$ is the union of the circles centered at origin, that intersect the set $(\cdot)$. We conjecture the assertion $(2.38)$ is valid in $C_{N D}(X, \mathcal{T} X)$. Note that the equality $s(M)=\omega(M)$ is an immediate consequence of (2.38).

\section{Description of the Spectrum}

In this section we will describe the spectrum $\sigma\left(e^{t M}\right)$ in the space $C_{N D}(X, \mathcal{T} X)$ under the assumptions of the previous section: $X$ is a compact Riemannian manifold with $\operatorname{dim} X \geq 3$ and the divergence-free vector field $v$ on $X$ generates the flow $\phi^{t}$ whose aperiodic points are dense in $X$. However, in fact, all the results of this section are valid provided $\operatorname{dim} X \geq 2$.

By Theorem 2.1 it suffices to determine $\sigma\left(e^{t M}\right)$ for a single value of $t$, say, for $t=1$. As a notational convenience, we define the bounded operator $T$ in $C(X, \mathcal{T} X)$ by $T=e^{M}$. For example, if $M=L$ is the Lie derivative in the direction $v$, then, for $x \in X$,

$$
(T u)(x)=D \phi\left(\phi^{-1} x\right) u\left(\phi^{-1} x\right) .
$$

Also, we let $T_{N D}:=T \mid C_{N D}(X, \mathcal{T} X)$ denote the restriction of $T$ to the subspace $C_{N D}(X, \mathcal{T} X)$.

We will prove that $\sigma\left(T_{N D}, C_{N D}(X, \mathcal{T} X)\right)$ is exactly one annulus, centered at the origin whose inner and outer boundaries are the boundaries of $\sigma(T, C(X, \mathcal{T} X))$. The proof is based on the following simple idea. We will 
show that both spectra are rotationally invariant and the approximate point spectra of $T$ and $T_{N D}$ coincide. Under the assumption that in the space of divergence-free vector fields $\sigma\left(T_{N D}, C_{N D}(X, \mathcal{T} X)\right)$ has a gap, we will extend the Riesz projection for $T_{N D}$ from $C_{N D}(X, \mathcal{T} X)$ to $C(X, \mathcal{T} X)$. To construct this extension, we will approximate a continuous vector field by a linear combination of locally supported divergence-free vector fields. The Riesz projection for $T_{N D}$ can be applied to each such divergence-free vector field and this extension turns out to be a Riesz projection for $T$ in $C(X, \mathcal{T} X)$. By Mather's theory this Riesz projection will be an operator of multiplication by a continuous matrix-valued function. This multiplication must preserve $C_{N D}(X, \mathcal{T} X)$, a contradiction.

To approximate a continuous vector field by a linear combination of locally supported divergence-free vector fields, we will need the following restricted form of Lemma 2.3:

Lemma 3.1 If $x^{0} \in X$ is a non periodic point of $v$ and if $z \in \mathcal{T}_{x^{0}} X$, then, for each pair $B, D$ of sufficiently small neighborhoods with $D \supset B \ni x^{0}$, there is a coordinate chart with coordinate functions $\left(x_{1}, \ldots, x_{n}\right)$ at $x^{0}$ containing $D$ and a vector field $f \in C_{N D}(X, \mathcal{T} X)$ with $\operatorname{supp} f \subset D$ such that the local representative of $f$ in $B$ is given by the constant vector field $\sum_{i=1}^{n} z_{i} \frac{\partial}{\partial x_{i}}$ whose components, $z_{i}$, are the components of the local representative of the vector $z$.

By a theorem of Mather [13] (see also [5, 12]), the spectrum $\sigma_{a p}(T)$ in $C(X, \mathcal{T} X)$ is invariant with respect to rotations about the origin in the complex plane. As a corollary of Theorem 2.1 we have the following two assertions. We note, that these two assertions were also proved in [21].

Corollary $3.2 \sigma_{a p}\left(T_{N D} ; C_{N D}(X, \mathcal{T} X)\right)$ is rotationally invariant.

Corollary $3.3 \sigma_{a p}(T ; C(X, \mathcal{T} X))=\sigma_{a p}\left(T_{N D} ; C_{N D}(X, \mathcal{T} X)\right)$.

Proof: In view of (2.8) and the fact that the spectra $T_{N D}$ and $T$ are rotationally invariant, the assertion will be proved as soon as we show the following proposition: If $1 \in \sigma_{\mathrm{ap}}(T ; C(X, \mathcal{T} X))$, then $1 \in \sigma_{\mathrm{ap}}\left(T_{N D} ; C_{N D}(X, \mathcal{T} X)\right)$. By the Spectral Inclusion Theorem (2.37), to prove this proposition it is enough to show that $0 \in \sigma_{\mathrm{ap}}\left(M_{N D} ; C_{N D}(X, \mathcal{T} X)\right)$ provided $1 \in \sigma_{\mathrm{ap}}(T)$ in $C(X, \mathcal{T} X))$. This is done in Lemma 2.7 . 
In accordance with [13] (see also $[5,12]$ ), the set $\sigma(T ; C(X, \mathcal{T} X))$ generally consists of several disjoint annuli centered at the origin. Let $r_{-}\left(\right.$resp., $\left.r_{+}\right)$denote the radius of the inner most (resp., outer most) circle in $\sigma(T ; C(X, \mathcal{T} X))$. By Corollary 3.3, we have $\sigma\left(T_{N D}, C_{N D}(X, \mathcal{T} X)\right) \subset\left\{z: r_{-} \leq|z| \leq r_{+}\right\}$. We will show the set $\sigma\left(T_{N D}, C_{N D}(X, \mathcal{T} X)\right)$ is exactly this annulus. This is the content of the next theorem.

Theorem 3.4 The spectrum $\sigma\left(T_{N D}, C_{N D}(X, \mathcal{T} X)\right)$ of $T$ in $C_{N D}(X, \mathcal{T} X)$ is the annulus $\left\{z: r_{-} \leq|z| \leq r_{+}\right\}$.

Proof: Suppose the theorem is not true, then there is a gap in the spectrum $\sigma\left(T_{N D}\right)$ in $C_{N D}(X, \mathcal{T} X)$. Without loss of generality, we can assume there is an annulus $\left\{z: r_{1} \leq|z| \leq r_{2}\right\}$ in the resolvent set of $T_{N D}$ containing the unit circle $\mathbb{T}$ and $r_{-} \leq r_{1}<1<r_{2} \leq r_{+}$. In this case, there is a Riesz projection $P=P_{N D}$ for the operator $T_{N D}$ in $C_{N D}(X, \mathcal{T} X)$ corresponding to the part of $\sigma\left(T_{N D}, C_{N D}(X, \mathcal{T} X)\right)$ that lies inside of the unit disc $\mathbb{D}$. In addition, there are positive constants $C_{1}, C_{2}$ such that

$$
\begin{aligned}
\operatorname{Im} P= & \left\{f \in C_{N D}(X, \mathcal{T} X):\right. \\
& \left.\left\|T^{n} f\right\|_{C(X, \mathcal{T} X)} \leq C_{1} r_{1}^{n}\|f\|_{C(X, \mathcal{T} X)}, n \in \mathbb{N}\right\} \\
\operatorname{Im}(I-P)= & \left\{f \in C_{N D}(X, \mathcal{T} X):\right. \\
& \left.\left\|T^{-n} f\right\|_{C(X, \mathcal{T} X)} \leq C_{2} r_{2}^{-n}\|f\|_{C(X, \mathcal{T} X)}, n \in \mathbb{N}\right\} .
\end{aligned}
$$

We will construct a projection $\mathcal{P}$ in $C(X, \mathcal{T} X)$ that commutes with $T$ and has the following additional properties:

$$
\sigma(T \mid \operatorname{Im} \mathcal{P}, C(X, \mathcal{T} X)) \subseteq \mathbb{D}, \quad \sigma\left([T \mid \operatorname{Im}(I-\mathcal{P})]^{-1}, C(X, \mathcal{T} X)\right) \subset \mathbb{D}
$$

In other words, the operator $T$ is hyperbolic in $C(X, \mathcal{T} X)$, that is,

$$
\sigma(T, C(X, \mathcal{T} X)) \cap \mathbb{T}=\emptyset
$$

and $\mathcal{P}$ is the Riesz projection for $T$ in $C(X, \mathcal{T} X)$. It follows, see [13] and [12], that the projection $\mathcal{P}$ has a form $\mathcal{P} f(x)=P_{C}(x) f(x)$, where $P_{C}: X \rightarrow$ $\operatorname{proj}\left(\mathcal{T}_{x} X\right)$ is a continuous projection-valued function.

Note, that $\mathcal{P}=P_{N D}$ on $C_{N D}(X, \mathcal{T} X)$. Hence, $\mathcal{P}$ maps $C_{N D}(X, \mathcal{T} X)$ into itself. We claim that this implies $\mathcal{P}$ is either the identity or the zero operator, in contradiction to the fact that $T$ is hyperbolic. To prove the 
claim, consider local (adapted) coordinates $\left(x_{1}, \ldots, x_{n}\right)$ so that the divergence operator is given as in (2.10). The projection $\mathcal{P}$ is represented by a matrix valued function with components $\mathcal{P}_{i j}(x)$. For each divergence-free vector field $u$, we then have

$$
0=\operatorname{div}(\mathcal{P} u)=\sum_{i, j} \frac{\partial \mathcal{P}_{i j}}{\partial x_{i}} u_{j}+\mathcal{P}_{i j} \frac{\partial u_{j}}{\partial x_{i}} .
$$

For each point $x$ in the coordinate chart and each index pair $i, j$ with $i \neq j$, there is a divergence-free vector field $u$ such that $u(x)=0$ and $\partial u_{j}(x) / \partial x_{i}=$ $\delta_{i j}$ where $\delta_{i j}$ is Kronecker's delta. With this choice of $u$, (3.41) shows $\mathcal{P}_{i j}=0$ for $i \neq j$. Using that fact that $\mathcal{P}$ is a projection, we have $\mathcal{P}(\mathcal{P}-I)=0$ and it follows that each diagonal element, $\mathcal{P}_{i i}(x)$, is either zero or one. Since $\mathcal{P}$ preserves all divergence-free vector fields, it is easy to see that all diagonal elements must then be equal and $\mathcal{P}$ is as required in the coordinate chart. The desired result follows by continuity and the connectivity of $X$.

We will construct the required projection $\mathcal{P}$ in the space $C(X, \mathcal{T} X)$.

Step 1. We introduce "step-functions" in $C(X, \mathcal{T} X)$.

Since $X$ is compact, there is a partition of unity $\left\{\rho_{k}\right\}_{k=1}^{K}$ with $K<\infty$, that is, for each integer $0<k \leq K$, the function $\rho_{k}: X \rightarrow[0,1]$ is continuous, and, for each $x \in X$,

$$
\begin{gathered}
\sum_{k=1}^{K} \rho_{k}(x)=1, \\
\operatorname{supp} \rho_{k} \backslash\left(\cup_{\ell \notin k} \operatorname{supp} \rho_{\ell}\right) \neq \emptyset .
\end{gathered}
$$

In particular, there is some $x_{k} \in \operatorname{supp} \rho_{k} \backslash \cup_{\ell \notin k} \operatorname{supp} \rho_{\ell}$ such that $\rho_{k}\left(x_{k}\right)=1$.

For each set of vectors $u_{1}, \ldots, u_{K}$, the vector field

$$
g(x)=\sum_{k=1}^{K} \rho_{k}(x) u_{k}
$$

is continuous, and $\|g\|_{C(X, \mathcal{T} X)}=\sup _{k}\left\|u_{k}\right\|$. Indeed, if $\left\|u_{k^{\prime}}\right\|=\sup _{k}\left\|u_{k}\right\|$, we can use (3.43) to choose $x_{0}$ such that $\rho_{k^{\prime}}\left(x_{0}\right)=1$ for some $x_{0} \in \operatorname{supp} \rho_{k^{\prime}} \backslash \cup_{k \notin k^{\prime}}$ $\operatorname{supp} \rho_{k}$. Then,

$$
\|g\| \geq\left\|g\left(x_{0}\right)\right\|=\left\|\sum_{k} \rho_{k}\left(x_{0}\right) u_{k}\right\|=\left\|\rho_{k^{\prime}}\left(x_{0}\right) u_{k^{\prime}}\right\|=\left\|u_{k^{\prime}}\right\|
$$


On the other hand, using (3.42),

$$
\|g\|=\max _{x \in X}\left\|\sum_{k} \rho_{k}(x) u_{k}\right\| \leq \max _{x \in X} \sum_{k} \rho_{k}(x)\left\|u_{k}\right\| \leq \sup _{k}\left\|u_{k}\right\| .
$$

It is also easy to see that the set $\mathfrak{G}$ of all such "step-functions" $g=\sum_{k} \rho_{k} u_{k}$ is dense in $C(X, \mathcal{T} X)$.

Step 2. We will define $\mathcal{P}$ for $g \in \mathfrak{G}$.

Suppose $g=\sum_{k} \rho_{k} u_{k} \in \mathfrak{G}$. Without loss of generality we can assume the partition of unity is so fine that Lemma 3.1 is applicable for each $k$. By this lemma, for each $k$, there is a section $f_{k} \in C_{N D}(X, \mathcal{T} X)$ such that $f_{k}(x)=u_{k}$ for $x \in \operatorname{supp} \rho_{k}$ and $\left\|f_{k}\right\|_{C}=\left\|u_{k}\right\|$. Then, for each $x \in X$,

$$
g(x)=\sum_{k} \rho_{k}(x) f_{k}(x), \quad f_{k} \in C_{N D}(X, \mathcal{T} X) .
$$

We define $\mathcal{P} g$ and $\mathcal{Q} g$ as follows:

$$
\mathcal{P} g(x)=\sum_{k} \rho_{k}(x)\left(P f_{k}\right)(x), \quad \mathcal{Q} g(x)=\sum_{k} \rho_{k}(x)\left[(I-P) f_{k}\right](x),
$$

where $P=P_{N D}$ is the Riesz projection for $T_{N D}$ in $C_{N D}(X, \mathcal{T} X)$. Formally, the decomposition $g=\mathcal{P} g+\mathcal{Q} g$, depends upon the choice of $f_{k}$. However, we will show that, in fact, the definition (3.45) does not depend on this choice and that $\mathcal{P}$ is a bounded linear operator on $\mathfrak{G}$. Once this is proved, the unique bounded linear extension of $\mathcal{P}$ to $C(X, \mathcal{T} X)$ is the desired projection.

Step 3. Define

$$
\begin{aligned}
& F_{+}:=\left\{f \in C(X, \mathcal{T} X): \lim _{n \rightarrow \infty}\left\|T^{n} f\right\|=0\right\} \\
& F_{-}:=\left\{f \in C(X, \mathcal{T} X): \lim _{n \rightarrow \infty}\left\|T^{-n} f\right\|=0\right\} .
\end{aligned}
$$

We will show that $F_{+} \cap F_{-}=\emptyset$.

Clearly, $F_{ \pm}$are linear (not necessarily closed) subspaces in $C(X, \mathcal{T} X)$. Assume $f \in F_{+} \cap F_{-}$and $f \neq 0$. We have

$$
\lim _{n \rightarrow \pm \infty}\left\|T^{n} f\right\|=\lim _{n \rightarrow \pm \infty} \max _{x}\|\Phi(x, n) f(x)\|=0 .
$$

In particular, there is some $x^{0} \in X$ such that $f\left(x^{0}\right) \neq 0$ and

$$
\sup _{n \in \mathbb{Z}}\left\|\Phi\left(x^{0}, n\right) f\left(x^{0}\right)\right\|<\infty .
$$


This implies (see [5] or [14]) that $\mathbb{T} \subset \sigma_{a p}(T, C(X, \mathcal{T} X)$ ). Thus, by Corollary 3.3, we have $\mathbb{T} \subset \sigma_{a p}\left(T_{N D}, C_{N D}(X, \mathcal{T} X)\right)$. But, this contradicts our assumption that $T_{N D}$ is hyperbolic in $C_{N D}(X, \mathcal{T} X)$.

Step 4. We show that $\mathcal{P}$ and $\mathcal{Q}$ are well-defined on $\mathfrak{G}$.

Suppose $g=\sum \rho_{k} u_{k} \in \mathfrak{G}$ and, for each $k$, the section $f_{k}$ is chosen as in (3.44). Also, define $g_{+}:=\mathcal{P} g$ and $g_{-}:=\mathcal{Q} g$. We will show $g_{ \pm} \in F_{ \pm}$.

Indeed, as $P f_{k} \in \operatorname{Im} P$, using the inequality (3.39), we have

$$
\begin{aligned}
\left\|T^{n} g_{+}\right\| & =\left\|\sum_{k} \rho_{k} \circ \phi^{n} T^{n} P f_{k}\right\|_{C(X, \mathcal{T} X)} \\
& \leq \sup _{k}\left\|T^{n} P f_{k}\right\|_{C(X, \mathcal{T} X)} \max _{x} \sum_{k} \rho_{k} \circ \phi^{n} \\
& \leq C_{1} r_{1}^{n} \sup _{k}\left\|P f_{k}\right\|_{C(X, \mathcal{T} X)} \leq C_{1} r_{1}^{n}\|P\| \sup _{k}\left\|f_{k}\right\|_{C(X, \mathcal{T} X)}= \\
& =C_{1} r_{1}^{n}\|P\| \sup _{k}\left\|u_{k}\right\|=C_{1} r_{1}^{n}\|P\| \cdot\|g\|_{C(X, \mathcal{T} X) .}
\end{aligned}
$$

In particular, $\lim _{n \rightarrow \infty}\left\|T^{n} g_{+}\right\|=0$ and $g_{+} \in F_{+}$. Similarly, using (3.40), we have

$$
\begin{aligned}
& \left\|T^{-n} g_{-}\right\|_{C(X, \mathcal{T} X)}=\left\|\sum_{k} \rho_{k} \circ \phi^{-n} T^{-n}(I-P) f_{k}\right\|_{C(X, \mathcal{T} X)} \\
& \leq \max _{x} \sum_{k} \rho_{k} \circ \phi^{-n}(x) \sup _{k}\left\|T^{-n}(I-P) f_{k}\right\|_{C(X, \mathcal{T} X)} \\
& \leq C_{2} r_{2}^{-n}\|I-P\| \sup _{k}\left\|f_{k}\right\|_{C(X, \mathcal{T} X)}=C_{2}\|I-P\| r_{2}^{-n}\|g\|_{C(X, \mathcal{T} X)} .
\end{aligned}
$$

This implies $\left\|T^{-n} g_{-}\right\| \rightarrow 0$ as $n \rightarrow \infty$ and $g_{-} \in F_{-}$.

By Step 3, we have $F_{+} \cap F_{-}=\{0\}$. Hence, $g_{ \pm}$, in the decomposition $g=g_{+}+g_{-}$with $g_{ \pm} \in F_{ \pm}$, are uniquely defined. If particular, the definition of $\mathcal{P} g$ and $\mathcal{Q} g$ in (3.45) does not depend on the choice of $f_{k}$.

Step 5. We extend $\mathcal{P}$ and $\mathcal{Q}$ from $\mathfrak{G}$ to $C(X, \mathcal{T} X)$.

From the calculations in Step 4 with $\tilde{C}_{1}:=C_{1}\|P\|$ and $\tilde{C}_{2}:=C_{2}\|I-P\|$, we have, for $n \in \mathbb{N}$, that

$$
\left\|T^{n} g_{+}\right\| C \leq \tilde{C}_{1} r_{1}^{n}\|g\|, \quad\left\|T^{-n} g_{-}\right\|_{C} \leq \tilde{C}_{2} r_{2}^{-n}\|g\| .
$$

These inequalities, for $n=0$, show that $\mathcal{P}$ and $\mathcal{Q}$ are bounded on $\mathfrak{G}$. To complete the proof we will show these operators are linear on $\mathfrak{G}$. 
Indeed, for

$$
g=\sum_{i=1}^{i_{0}} \rho_{i} u_{i} \text { and } \tilde{g}=\sum_{j=1}^{j_{0}} \tilde{\rho}_{j} \tilde{u}_{j}
$$

there are $f_{i}, \tilde{f}_{j} \in C_{N D}(X, \mathcal{T} X)$ such that $g=\sum \rho_{i} f_{i}$ and $\tilde{g}=\sum \tilde{\rho}_{j} \tilde{f}_{j}$. We define $f_{i j}=f_{i}$ and $\tilde{f}_{i j}=\tilde{f}_{j}$ for $i=1, \ldots, i_{0}, j=1, \ldots, j_{0}$, and use (3.42) to obtain:

$$
g=\sum_{i, j} \rho_{i} \tilde{\rho}_{j} f_{i j}, \quad \tilde{g}=\sum_{i, j} \rho_{i} \tilde{\rho}_{j} \tilde{f}_{i j}
$$

Then, (3.45) gives:

$$
\mathcal{P}(g+\tilde{g})=\sum_{i, j} \rho_{i} \tilde{\rho}_{j}\left(P f_{i j}+P \tilde{f}_{i j}\right)=\mathcal{P} g+\mathcal{P} \tilde{g},
$$

as required.

From this theorem and Theorem 2.1 we conclude, that the spectrum $\sigma(M)$ in the space $C_{N D}(X, \mathcal{T} X)$ of divergence-free vector fields is exactly one vertical strip:

Corollary $3.5 \sigma\left(M ; C_{N D}(X, \mathcal{T} X)\right)=\left\{z: \ln r_{-} \leq \operatorname{Re} z \leq \ln r_{+}\right\}$.

Our next goal is to characterize the spectra $\sigma\left(T_{N D} ; C_{N D}(X, \mathcal{T} X)\right)$ and $\sigma\left(M_{N D} ; C_{N D}(X, \mathcal{T} X)\right)$ via the exact Lyapunov exponents for the cocycle $\Phi(x, t)$ with respect to the set of $\phi^{t}$-ergodic measures $\nu \in \mathcal{E}$. Recall that, by the Multiplicative Ergodic Theorem [17], for each ergodic measure $\nu \in \mathcal{E}$, there exists a set $X_{\nu} \subset X$ with $\nu\left(X_{\nu}\right)=1$ such that for each $x \in X$ and $u \in \mathcal{T}_{x} X$ there exist exact Lyapunov exponents

$$
\lambda_{\nu}(x, u)=\lim _{t \rightarrow \pm \infty} \frac{1}{t} \ln \|\Phi(x, t) u\| .
$$

For each $\nu$, there may exist $n^{\prime}=n^{\prime}(\nu) \leq n$ different Lyapunov exponents; we will denote them by $\lambda_{\nu}^{1}>\lambda_{\nu}^{2}>\ldots>\lambda_{\nu}^{n^{\prime}}$.

Corollary 3.6 The boundary circles of the spectrum $\sigma\left(T_{N D} ; C_{N D}(X, \mathcal{T} X)\right)$ and the spectrum $\sigma\left(M_{N D} ; C_{N D}(X, \mathcal{T} X)\right)$ are given by

$$
\ln r_{+}=\sup \left\{\lambda_{\nu}^{1}: \nu \in \mathcal{E}\right\}, \quad \ln r_{-}=\inf \left\{\lambda_{\nu}^{n^{\prime}}: \nu \in \mathcal{E}\right\} .
$$

There exist measures $\nu_{+}$and $\nu_{-}$and exact Lyapunov exponents $\lambda_{\nu_{+}}\left(x_{+}, u_{+}\right)$ and $\lambda_{\nu_{-}}\left(x_{-}, u_{-}\right)$, such that the sup and inf above are attained. 
Proof: For the boundaries $r_{ \pm}$of the spectrum $\sigma(T ; C(X, \mathcal{T} X))$ in $C(X, \mathcal{T} X)$ these formulas were obtained in [12] (see also [18]).

Remark 3.7. The absence of nontrivial spectral components of $\sigma(L)$ for the space $C_{N D}(X, \mathcal{T} X)$ leads to the following observation. Consider a situation when $L$ acts in the space $C(X, \mathcal{T} X)$. After some inessential modifications, we can obtain a dichotomic (no spectrum on $i \mathbb{R}$ ) operator $L$. For example, starting with an Anosov flow, as usual in Mather's theory, such an operator can be obtained by "moding out" the direction of the flow.

We note that "moding out" the direction of the flow does not change $\sigma(L)$. The reason is that the spectrum of $L$ on the direct sum of the quotient space $C_{N D}(X, \mathcal{T} X /[v])$ and the space of sections generated by $v$ is the union of the respective spectra. An element of the second space must be a divergence-free vector field of the form $\alpha v$ where $\alpha$ is a function on the manifold. This implies $\operatorname{grad} \alpha=0$ so that $\alpha$ is constant along the trajectories of $v$. But, then $L \alpha v=0$ and the spectrum of $L$ on this subspace is $\{0\}$. Since $\sigma\left(L_{N D} ; C_{N D}(X, \mathcal{T} X)\right)$ is invariant with respect to vertical translations in the complex plane, the entire imaginary axis must be in $\sigma\left(L_{N D}, C_{N D}(X, \mathcal{T} X)\right)$. All the points except the origin must then be in the spectrum of $L$ restricted to the quotient space. But the spectrum of $L$ is closed, thus the origin is already in the spectrum on the quotient. In particular, the spectrum of the quotient is the same as the spectrum on the original space.

Going back to the kinematic dynamo equations (1.1), we make the following concluding remark.

Remark 3.8. Recall (see, e.g., $[1,2,3]$ ) that kinematic dynamo is called "fast" provided $\lim \sup _{\varepsilon \rightarrow \infty} \omega\left(L_{\varepsilon}\right)$ is positive. M. Vishik [23] gave the following sufficient condition for the non-existence of a fast kinematic dynamo: Define the Lyapunov numbers

$$
\bar{\lambda}(x, u)=\limsup _{t \rightarrow \infty} \frac{1}{t} \ln \left\|D \phi^{t}(x) u\right\| .
$$

If

$$
\sup \{\bar{\lambda}(x, u): x \in X, u \in \mathcal{T} X\} \leq 0,
$$

then there is no fast kinematic dynamo. The fact that the spectral bound $\omega(L)$ is less than or equal to the supremum in (3.47), see Remark 2.10, is used in [23]. Therefore, in view of [23], our Corollary 3.6 gives an alternate form of the sufficient condition for no fast kinematic dynamo. 


\section{Acknowledgement}

We thank Misha Vishik for several very helpful conversations.

\section{References}

[1] V. I. Arnold, Some remarks on the antidynamo theorem, Moscow University Mathem. Bull., 6 (1982) 50-57.

[2] V. I. Arnold, Ya. B. Zel'dovich, A. A. Rasumaikin, and D. D. Sokolov, Magnetic field in a stationary flow with stretching in Riemannian space, Sov. Phys. JETP, 54 (6) (1981) 1083-1086.

[3] B. J. Bayly and S. Childress, Fast-dynamo action in unsteady flows and maps in three dimensions, Phy. Rev. Let. 59 (14) (1987) 1573-1576.

[4] C. Chicone, and Y. Latushkin, Quadratic Lyapunov functions for linear skew-product flows and weighted composition operators, J. Int. Diff. Eqns., to appear.

[5] C. Chicone and R. Swanson, Spectral theory for linearization of dynamical systems, J. Diff. Eqns., 40 (1981) 155-167.

[6] S. Friedlander and M. Vishik, Dynamo theory methods for hydrodynamic stability, J. Math. Pures Appl. 72 (1993) 145-180.

[7] M. Hirsch, C. Pugh, and M. Shub, Invariant Manifolds, Lect. Notes Math. 583 (1977).

[8] R. Johnson, Analyticity of spectral subbundles, J. Diff. Eqns., 35 (1980) 366-387.

[9] Y. Latushkin and S. Montgomery-Smith, Evolutionary semigroups and Lyapunov theorems in Banach spaces, J. Funct. Anal., to appear.

[10] Y. Latushkin and S. Montgomery-Smith, Lyapunov theorems for Banach spaces, Bull. AMS 31 (1) (1994) 44-49. 
[11] Y. Latushkin, S. Montgomery-Smith and T. Randolph, Evolutionary semigroups and dichotomy of linear skew-product flows on locally compact spaces with Banach fibres, in preparation.

[12] Y. Latushkin and A. M. Stepin, Weighted composition operators and linear extensions of dynamical systems, Russian Math. Surveys, 46, no. 2, (1992) 95-165.

[13] J. Mather, Characterization of Anosov diffeomorphisms, Indag. Math., 30 (1968) 479-483.

[14] R. Manẽ, Quasi-Anosov diffeomorphisms and hyperbolic manifolds, Trans. Amer. Math. Soc., 229 (1977) 351-370.

[15] H. K. Moffatt, Magnetic Field Generation in Electrically Conducting Fluids, Cambridge Univ. Press, Cambridge, 1978.

[16] S. A. Molchanov, A. A. Ruzmaikin, and D. D. Sokolov, Kinematic dynamo in random flow, Sov. Phys. Usp. 28 (4) (1985) 307-327.

[17] V. I. Oseledec, A multiplicative ergodic theorem: Lyapunov characteristic numbers for dynamical systems, Trans. Moscow Math. Soc., 19 (1968) 197-231.

[18] V. I. Oseledec, $\Lambda$-entropy and the anti-dynamo theorem, In: Proc. 6th Intern. Symp. on Information Theory, Part III, Tashkent, (1984) 162163.

[19] A. Pazy, Semigroups of Linear Operators and Applications to Partial Differential Equations, Springer-Verlag, N.Y./Berlin, 1983.

[20] Ya. B. Pesin, Hyperbolic theory, in: Encyclop. Math. Sci. Dynamical Systems 2 (1988).

[21] R. de la Llave, Hyperbolic dynamical systems and generation of magnetic fields by perfectly conducting fluids, Preprint, 1993.

[22] R. Rau, Hyperbolic linear skew-product semiflows, subm. to J. Diff. Eqns.

[23] M. M. Vishik, Magnetic field generation by the motion of a highly conducting fluid, Geophys. Astrophys. Fluid Dynamics, 48 (1989) 151-167. 